\title{
Effects of Resistance Training of Peripheral Muscles Versus Respiratory Muscles in Older Adults With Sarcopenia Who are Institutionalized: A Randomized Controlled Trial
}

\author{
Maria À. Cebrià i Iranzo, Mercè Balasch-Bernat, María Á. Tortosa-Chuliá, and Sebastià Balasch-Parisi
}

\begin{abstract}
This study compares the effects of two resistance training programs in peripheral and respiratory musculature on muscle mass and strength and physical performance and identifies the appropriate muscle mass parameter for assessing the intervention effects. Thirty-seven institutionalized older Spanish adults with sarcopenia were analyzed: control group $(n=17)$, respiratory muscle training group $(n=9)$, and peripheral muscle training group $(n=11)$. Measured outcomes were appendicular skeletal muscle mass (ASM/height ${ }^{2}$, ASM/weight, and ASM/BMI), isometric knee extension, arm flexion and handgrip strength, maximal inspiratory and expiratory pressures, and gait speed pre- and postintervention. Trained groups participated in a 12-week program and improved in maximum static inspiratory pressure, maximum static expiratory pressure, knee extension, and arm flexion $(p<.05)$, whereas nonsignificant changes were found in gait speed and ASM indexes pre- and postintervention in the three groups. In conclusion, resistance training improved skeletal muscle strength in the studied population, and any ASM index was found to be appropriate for detecting changes after physical interventions.
\end{abstract}

Keywords: aging, exercise training, muscle strength, nursing homes, prevention

The presence of comorbidity and other factors such as physical inactivity in older people favor the onset of sarcopenia (Cruz-Jentoft et al., 2010). The prevalence of this geriatric syndrome in older adults who are institutionalized is around 14-33\% in Europe (Cruz-Jentoft et al., 2014), and even higher in Spain (37\%) (Salvà et al., 2016).

The European Working Group on Sarcopenia in Older People (EWGSOP) defines this syndrome as a gradual and widespread loss of skeletal muscle mass and strength. As a result, mobility disorders appear, such as an increased risk of falls and fractures, impaired ability to perform daily living activities, loss of independence, a worsening of comorbidity, and an increased risk of death (CruzJentoft et al., 2010). Recently, the International Working Group on Sarcopenia (IWGSP) and Asian Working Group for Sarcopenia (AWGSP) shared this definition, but both groups suggested working toward a more appropriate selection of diagnostic cutoff values (Chen et al., 2014; Wei-Ju, Li-Kuo, Li-Ning, Ming-Hsien, \& Liang-Kung, 2013). In this respect, recent studies (Ethgen, Beaudart, Buekinx, Bruyere, \& Reginster, 2016; Kim, Jang, \& Lim, 2016; Meng et al., 2015) emphasize the need to personalize the cutoff values according to the characteristics of the studied population (anthropometrics, ethnicity, and clinical setting).

Previous literature points out that this loss affects the skeletal muscles involved in both mobility (limb or peripheral muscles, PMs) and ventilation (respiratory muscles, RMs). Enright, Kronmal, Manolio, Schenker, and Hyatt (1994) and Neder, Andreoni, Lerario, and Nery (1999) corroborate the association between the strength of the PMs and RMs. In studies of older adults who are institutionalized,

Cebrià i Iranzo and Balasch-Bernat are with the Dept. of Physiotherapy, Universitat de València, Valencia, Spain. Cebrià i Iranzo is also with Cardiopulmonary Rehabilitation Dept., University Hospital "La Fe," Valencia, Spain. Tortosa-Chuliá is with the Dept. of Applied Economics, Universitat de València, Valencia, Spain. Balasch-Parisi is with the Dept. of Applied Statistics and Operational Research and Quality, Universitat Polytècnica de València, Valencia, Spain. Address author correspondence to Maria À. Cebrià i Iranzo at angeles.cebria@uv.es. the prevalence of decreased strength of the PMs and RMs is clear (Cruz-Jentoft et al., 2010; Simões, Castello, Auad, Dionísio, \& Mazzonetto, 2009; Simões, Dias, Marinho, Pinto, \& Britto, 2010). Authors such as Newman et al. (2003) suggest that during aging the decline in lower limb muscle strength is greater than in upper limbs and is associated with lower gait speed and increased risk of disability. Moreover, dysfunction of the RMs is accompanied by reduced tolerance associated with basic activities of daily living and in some extreme cases with respiratory failure (Bahat et al., 2014).

In addition, most systematic reviews and meta-analyses of sarcopenia in older adults (Beaudart, Zaaria, Pasleau, Reginster, \& Bruyère, 2017; Cruz-Jentoft et al., 2014) suggest that exercise interventions (mainly resistance exercises) can improve muscle strength and physical performance, with the majority of these studies being carried out in older adults living in the community. In this respect, it is worthwhile to extend the research to older adult groups living in other settings, such as hospitals and nursing homes. Currently, studies show a high prevalence of sarcopenia in older adults residing in nursing homes (Henwood, Keogh, Reid, Jordan, \& Senior, 2014; Landi et al., 2012), and initial results regarding resistance training and sarcopenia in nursing care facilities are emerging. These are mainly focused on the improvement in the strength of only the PMs (Hassan et al., 2016) or of only the RMs (Cebrià i Iranzo, Arnall, Igual Camacho, Tomás, \& Meléndez, 2013), but there is a need for studies that compare the resistance training interventions.

It is assumed that the physical intervention in the older adults who are institutionalized is accompanied by fewer benefits (CruzJentoft et al., 2011). The clinical novelty of this study is that it shows resistance exercise interventions in nursing homes may improve, or at least control, the deterioration that sarcopenia entails in older adults, as well as reduce the risk of mortality associated with muscle strength (Newman et al., 2006). At the same time, it highlights the usefulness of this exercise intervention as a routine in nursing home planning. 
In light of the above, and considering the importance of preventing sarcopenia effects in institutionalized older people, the main objective of this study was to compare the effects of two resistance training programs in peripheral and respiratory musculature on muscle mass and strength and physical performance. A secondary objective was to compare and identify what muscle mass parameters are most appropriate for assessing the effects of resistance training programs in older adults who are institutionalized in Spain. We hypothesized that resistance training would improve muscle mass and strength and physical performance in older adults with sarcopenia, although these effects would depend on the specific resistance training program (PM or RM training).

\section{Methods}

\section{Study Design}

This study was designed as a parallel-group randomized controlled trial in which 81 institutionalized older Spanish adults with sarcopenia were randomized in a single sequence (simple randomization) to one of three balanced groups: one control group (CG) and two resistance training groups (peripheral muscle training group [PMTG] and respiratory muscle training group [RMTG]). A flow diagram (Figure 1) describes participant eligibility, randomization, participants lost to follow-up, and data analysis. A statistician who had no contact with the participants and/or the health-care professionals who undertook the measurements and intervention performed the random allocation sequence (random number generator in SPSS; SPSS, Inc., Chicago, IL). The three groups were followed in the same way, and the only difference between them was the received care. CG participants obtained standard treatment and were asked to maintain their usual care and daily life activities at the nursing home (lying down, sitting, and walking short distances between rooms). Participants of the intervention groups (PMTG and RMTG) also carried out a resistance training program for 12 weeks.

The Universitat de València Ethics Committee for Human Research (H1382044172319) approved this study. All participants were informed of the risks and benefits and agreed to participate by signing a consent form. This study was performed between 2013 and 2016 and has been registered at ClinicalTrials.gov (NCT ID: NCT02120586).

\section{Participants}

The sample was made up of all eligible older adults from four nursing homes located in Valencia, Spain. The nursing homes involved met similar criteria in relation to classification of the participants and had similar professional health services. Participants met the following inclusion criteria: (a) older than 65 years; (b) clinically stable for 2 months before the study; and (c) compliance with the sarcopenia diagnostic criteria proposed by Tyrovolas et al. (2015) which include (a) skeletal muscle mass index $(\mathrm{SMI}=$ appendicular skeletal muscle mass [ASM]/body mass index [BMI]) with cutoff points for the Spanish population ( $\leq 0.93$ for male and $\leq 0.57$ for female) and (b) gait speed with cutoff



Figure 1 - Flow diagram: $\mathrm{CG}=$ control group; RMTG = respiratory muscle training group; PMTG= peripheral muscle training group. 
points according to sex, height, and age (between 0.95 and $0.66 \mathrm{~m} / \mathrm{s}$ for male and 0.80 and $0.48 \mathrm{~m} / \mathrm{s}$ for female). Exclusion criteria were as follows: (a) clinical situations (signs and/or symptoms) that might interfere in proper performance of assessment and/or training protocols, for example, presence of edema or severe disorder of hydration status that could interfere with bioelectrical impedance analysis (Rubbieri, Mossello, \& Di Bari, 2014), malnutrition, muscle or joint pain, tremor or dyspnea at rest, recent fracture or surgery; (b) a terminal disease diagnosis; or (c) moderate or severe cognitive deterioration that might affect proper collaboration (Mini-Mental State Examination score $\leq 20$ points) (Lobo, Saz, \& Marcos, 2002).

\section{Measurements}

Participants were assessed at enrollment (T1), after a 2-week familiarization (T2, baseline or preintervention), and at 12 weeks (T3, postintervention). The reason for repeating two assessments (T1 and T2) within a short period of time was to avoid possible learning effect. These assessments were conducted by researchers who were trained in the procedures by an expert before the data collection period and were blind to the purpose of the study and the participants' group assignments. Tests were performed over two consecutive days to avoid participant fatigue and obtain the highest value.

\section{Outcomes and Procedures}

Measurements at each time point (T1-T3) showed the following outcomes:

Skeletal muscle mass. Bioimpedance analysis technique was used to assess muscle mass (in kg) using a Bodystat ${ }^{\circledR}$ 1500MDD (Bodystat Ltd., Douglas, United Kingdom). Measurements were obtained under recommended standard conditions (National Institutes of Health, 1996): participants in supine position on the bed, on waking up and with an empty stomach, and upper and lower limbs of the predominant side abducted at an at least $30^{\circ}$ angle from the median line, with a controlled bedroom temperature of $25{ }^{\circ} \mathrm{C}$. Equipment calibration, individual data collection (sex, age, height, and weight), and skin preparation for the placement of the adhesive electrodes on the wrist and the ankle of the predominant side had been accomplished prior to the measurements. According to Meng et al. (2015) and Kim et al. (2016), the skeletal mass index was defined as ASM, after being adjusted in different ways: height (ASM/height ${ }^{2}$ ), weight (ASM/weight), or BMI (ASM/BMI).

PM strength. Maximal isometric muscle strength (in $\mathrm{kg}$ ) was assessed with the dynamometer MicroFet2® (Hoggan Health Industries, West Jordan, UT) on the predominant side for three consecutive trials with an at least 1-min resting period in between. Prior to strength testing, a 5-min warm-up for the upper and lower limbs on the Monark $®$ Rehab Ergometer Trainer (Monark 881E; Monark Exercise, Vansbro, Sweden) was performed. Participants remained in a seated position with the muscle to be evaluated in relative shortened position, maintaining a maximal and sustained contraction for at least $3 \mathrm{~s}$. The movements tested were knee extension (quadriceps femoris) and elbow flexion (biceps brachii) (Bean et al., 2002).

The maximal handgrip strength (in $\mathrm{kg}$ ) was measured with a hydraulic hand dynamometer Jamar Plus+® (Patterson Medical, Sammons Preston, Bolingbrook, IL). Participants remained seated, the dynamometer in the dominant hand, with the elbow locked at $90^{\circ}$ and held at the side. Predicted values (in \%) for handgrip strength were also registered (Luna-Heredia, Martín-Peña, \& RuizGaliana, 2005). Three consecutive measurements were made on the predominant side upper limb with a between-measurement interval of $1 \mathrm{~min}$.

RM strength. Maximum static inspiratory pressure (MIP, $\mathrm{cmH}_{2} \mathrm{O}$ ) and maximum static expiratory pressure $\left(\mathrm{MEP}, \mathrm{cmH}_{2} \mathrm{O}\right)$ were measured using a MicroRPM $®$ (respiratory pressure meter; CareFusion, Höechberg, Germany) according to the Black and Hyatt (1969) technique. These strength measurements followed the standard guidelines established by the American Thoracic Society/ European Respiratory Society (2002). Predicted values (in \%) for both MIP and MEP were also registered using the reference values reported by Enright et al. (1994).

For PM and RM strength data, the highest value was recorded.

RM endurance. The maximal voluntary ventilation test (MVV, L/min) was performed following the standard guidelines established by the American Thoracic Society/European Respiratory Society (2002), using a portable Jaeger ${ }^{\circledR}$ spirometer is Jaeger Flow Screen VIASYS Healthcare GmbBH (Hoechberg, Germany) as referred to above.

Physical performance. Maximal gait speed (m/s) was collected as participants walked as fast as they safely could on a marked 14-m walkway. Participants started and finished walking $2 \mathrm{~m}$ before and after the walkway to ensure that they reached a steady-state gait speed across the middle $10 \mathrm{~m}$ (Tilson et al., 2010). Each participant performed two consecutive trials with a 1-min resting period in between, and the highest value was recorded. Pulse oximetry was registered during the test, and the participants were asked about their subjective effort perception using the Borg CR10 Scale (Borg, 2004).

\section{Interventions}

Both intervention groups participated in a 12-week training program, three times a week on alternate days, in groups of eight to 10 participants. All training sessions were supervised by two physiotherapists not involved in the assessments (T1-T3). Prior to this, familiarization consisted of six sessions to guarantee appropriate preparation. This was performed with a low training workload (5-10\% of maximal muscle strength [kg] for PMTG and 7-9 $\mathrm{cmH}_{2} \mathrm{O}$ for RMTG). As some of the participants could not remain standing, the training was performed in a sitting position. Each session was structured in four phases: (a) recording of resting blood pressure, heart frequency, and oxygen saturation $\left(\mathrm{SpO}_{2}\right)$; (b) an initial 5-min warm-up; (c) resistance exercises with workload for 20-30 min; and (d) a final 5-min cooldown to recover baseline condition. Individual training diaries were kept on the progress of each participant, documenting the increases in training workload and as well recording perceived exertional efforts (Borg CR10 Scale) and any complaints related to the training program reported by the participants. To ensure that each participant was training with the appropriate workload, a monthly measurement was performed. Participants who attended less than $80 \%$ of the sessions in both programs were dropped from the analyses.

PM training. PM training consisted of 10 isotonic resistance exercises with 12 repetitions of each one, completing the maximum mobility at a slow speed, working both concentric and eccentric phases. Between each isotonic resistance exercise there was $2 \mathrm{~min}$ of recovery. Resistance exercises were performed with a workload adjusted to 40-60\% of maximal isometric muscle strength (in $\mathrm{kg}$ ), 
except when participants did not complete the maximum mobility, in which case the workload was reduced. Exercises were performed with dumbbells and ankle or wrist weights: four lower limb exercises (ankle flexion/extension, knee extension, and hip flexion/ abduction/adduction) and six upper limb exercises (handgrip, wrist flexion/extension, forearm pronation/supination, elbow flexion/ extension, and shoulder flexion/extension/adduction/abduction).

RM training. RM training was carried out using a Threshold Inspiratory Muscle Trainer device (Respironics $®$ Health Scan Inc., Cedar Grove, NJ) with a working range of 7-41 $\mathrm{cmH}_{2} \mathrm{O}$. An interval-based inspiratory muscle training program was performed consisting of seven 2-min cycles of loaded breathing interspersed with 1-min rest periods between cycles (Hill, Cecins, Eastwood, \& Jenkins, 2010). The workload was adjusted to 40-60\% of MIP (in $\mathrm{cmH}_{2} \mathrm{O}$ ), except when participants did not complete the interval training program, in which case the workload was reduced. The physiotherapists monitored the participants throughout the training session with a pulse oximeter $\left(\mathrm{SpO}_{2}\right.$ and heart frequency). In this way, each participant adopted her or his own comfortable respiratory rate, avoiding undesirable desaturations and/or heart rate increases, as well as symptoms of hyperventilation.

\section{Statistical Analyses}

A priori sample size calculations were obtained with the statistical program G*Power 3.1 (Faul, Erdfelder, Lang, \& Buchner, 2007), with an expected effect size $(f)$ from medium to large, $\alpha$ of .05 , and statistical power of .80 . This resulted in an a priori total sample size of 72 participants, or 24 per group.

Analyses were conducted using the statistical software Statgraphics Centurion XVI.II (Statgraphics Technologies, Inc., The Plains, VA). All data were checked for outliers, normal distributions (Shapiro-Wilk test), and homogeneity of variance (Levene's test).

Descriptive statistics were conducted for all variables at preintervention (T2). Moreover, preintervention differences of the variables between groups were examined to test whether any clinical or anthropometric variable could be included as a covariate in the analysis of variance (ANOVA). For this purpose, one-way ANOVA was conducted for all continuous variables and chi-square tests were used for categorical variables.

Treatment effects between the three groups (PMTG, RMTG, and $\mathrm{CG}$ ) were determined using one-way ANOVA on change from preintervention, defined as postintervention (T3) minus preintervention (T2) values for each variable. Change analysis was used to offset possible differences of participants at preintervention and to minimize group data variability. Within-group differences were assessed using paired $t$ tests to compare mean change values. Between-group differences were calculated using a three-way ANOVA, with group cardiovascular disease and endocrine disease as factors. Cardiovascular disease and endocrine disease were included as (categorical) covariates to eliminate the variability attributable to these variables. Post hoc analyses were performed using Tukey's highly significant difference test when a significant effect was found. Statistical significance was set at $p<.05$ for all tests.

\section{Results}

\section{Participants}

The analyzed sample was made up of 37 older adults. The flow diagram (Figure 1) shows 44 dropouts, the main reasons being deaths, exacerbations, attendance of fewer than $80 \%$ of the sessions in trained groups, and withdrawals (i.e., changing to another nursing home, falls, personal reasons, or complaints). Deaths during the study development were as a result of the participants' illnesses and not from factors related to the measurements and/or training programs. Main complaints reported by participants occurred within the first 2 weeks of training, and the most common were difficulty keeping the lips constrained in the mouthpieces of the Inspiratory Muscle Training device and nasal discomfort from noseclip placement during the session, in the RMTG; and mild muscular discomfort and generalized fatigue that disappeared between sessions, in the PMTG.

\section{Descriptive}

Baseline characteristics. Preintervention characteristics of the three groups are presented in Table 1. Initially, the three groups did not show differences, except for cardiovascular $(p=.02)$ and endocrine ( $p=.04$ ) diseases. PMTG participants presented a higher number of cardiovascular and endocrine dysfunctions than the other two groups.

\section{Effects of the Training Programs}

Results from the within-group and between-group effects over the main outcomes are summarized in Table 2. No effects were found from endocrine and cardiovascular disease in any variables between pre- and postintervention in the three groups.

Skeletal muscle mass. No significant changes were detected in any of the skeletal muscle mass measures (ASM /height ${ }^{2}$, ASM/ weight, and ASM/BMI) between pre- and postintervention in the three groups.

PM strength. A significant decrease occurred from pre- to postintervention in CG for quadriceps femoris, $13.1 \%$; $t(11)=-2.33$; $p=.040$, and biceps brachii, $23.8 \% ; t(11)=-2.84 ; p=.016$. Results for the PMTG showed a significant increase in quadriceps femoris, $51.9 \% ; t(6)=3.66 ; p=.011$, and biceps brachii, $17.4 \%$; $t(7)=2.40 ; p=.048$. For the RMTG, quadriceps femoris showed an increasing trend, $9.1 \% ; t(7)=0.18 ; p=.859$, whereas biceps brachii showed a significant decrease, $16.7 \% ; t(8)=-2.34 ; p=.048$.

Moreover, significant differences in PM strength between groups were also observed for both quadriceps femoris $(p=.009)$ and biceps brachii $(p=.003)$. Post hoc analysis of quadriceps femoris strength showed that increases were greater in PMTG than in CG participants. For biceps brachii strength, changes for the PMTG were greater than changes for the other two groups.

No significant changes were seen in dominant handgrip strength values between pre- and postintervention. The same effects were detected for handgrip strength predicted values.

$\boldsymbol{R} \boldsymbol{M}$ strength. The within-group ANOVA revealed significant differences in MIP and MEP values for CG between pre- and postintervention. CG subjects decreased their MIP and MEP with a mean of $8.2 \%, t(12)=-2.62 ; p=.022$, and $15.6 \%, t(12)=-2.32 ; p=.039$, respectively. PMTG showed a nonsignificant ascending trend of $13.5 \%$ in MIP, $t(8)=1.86 ; p=.100$, and of $3.9 \%$ in MEP, $t(8)=0.63$; $p=.548$. Likewise, changes from pre- to postintervention in these variables for RMTG were found to be statistically nonsignificant.

Analysis of variance comparisons between groups revealed significant differences for MIP $(p=.007)$ and MEP $(p=.040)$. Post hoc analysis showed greater changes for PMTG and RMTG than for CG. Similar effects were detected for MIP and MEP predicted values. 
Table 1 Baseline Characteristics of Studied Groups

\begin{tabular}{|c|c|c|c|c|}
\hline & CG $(n=17)$ & RMTG $(n=9)$ & PMTG $(n=11)$ & $p$ \\
\hline \multicolumn{5}{|l|}{ Anthropometrics } \\
\hline gender, M/F (\%) & $29 / 71$ & $44 / 56$ & $18 / 82$ & .44 \\
\hline age (years) & $81.2 \pm 5.4$ & $87.1 \pm 3.8$ & $82.6 \pm 9.1$ & .10 \\
\hline weight (kg) & $70.1 \pm 14.6$ & $73.6 \pm 19.8$ & $71.8 \pm 16.4$ & .87 \\
\hline height $(\mathrm{cm})$ & $149.8 \pm 8.5$ & $151.8 \pm 12.2$ & $151.7 \pm 8.2$ & .82 \\
\hline BMI $\left(\mathrm{kg} / \mathrm{m}^{2}\right)$ & $31.2 \pm 5.9$ & $32.0 \pm 8.3$ & $31.4 \pm 7.5$ & .96 \\
\hline \multicolumn{5}{|l|}{ Medical history (\%) } \\
\hline respiratory disease, no/yes & $88 / 13$ & $67 / 33$ & $91 / 9$ & .29 \\
\hline cardiovascular disease, no/yes & $19 / 81$ & $56 / 44$ & $73 / 27$ & $.02 *$ \\
\hline endocrine disease, no/yes & $38 / 63$ & $33 / 67$ & $82 / 18$ & $.04 *$ \\
\hline neurological disease, no/yes & $31 / 69$ & $78 / 22$ & $64 / 36$ & .06 \\
\hline muscular disease, no/yes & $100 / 0$ & $100 / 0$ & $100 / 0$ & - \\
\hline skeletal disease, no/yes & $44 / 56$ & $44 / 56$ & $36 / 64$ & .91 \\
\hline other diseases, no/yes & $75 / 25$ & $100 / 0$ & $55 / 45$ & .07 \\
\hline \multicolumn{5}{|l|}{ Sarcopenia criteria } \\
\hline \multirow[t]{3}{*}{$\mathrm{ASM} / \mathrm{ht}^{2}\left(\mathrm{~kg} / \mathrm{m}^{2}\right)$} & $8.5 \pm 1.9$ & $8.9 \pm 2.0$ & $8.3 \pm 2.2$ & .85 \\
\hline & M: $10.34 \pm 0.93$ & M: $9.59 \pm 1.51$ & M: $9.86 \pm 0.87$ & M: .64 \\
\hline & F: $7.76 \pm 1.70$ & $F: 8.28 \pm 2.29$ & $\mathrm{~F}: 7.97 \pm 2.28$ & F: .89 \\
\hline \multirow[t]{3}{*}{ ASM/wt (\%) } & $0.3 \pm 0.1$ & $0.3 \pm 0.1$ & $0.3 \pm 0.1$ & .83 \\
\hline & M: $0.36 \pm 0.02$ & M: $0.35 \pm 0.03$ & M: $0.35 \pm 0.01$ & M: .82 \\
\hline & $F: 0.24 \pm 0.01$ & $F: 0.23 \pm 0.02$ & $F: 0.25 \pm 0.01$ & $\mathrm{~F}: .12$ \\
\hline \multirow[t]{3}{*}{$\operatorname{ASM} / \mathrm{BMI}\left(\mathrm{m}^{2}\right)$} & $0.6 \pm 0.2$ & $0.7 \pm 0.2$ & $0.6 \pm 0.2$ & .85 \\
\hline & M: $0.91 \pm 0.06$ & M: $0.93 \pm 0.05$ & M: $0.93 \pm 0.02$ & M: .95 \\
\hline & $\mathrm{F}: 0.51 \pm 0.05$ & $F: 0.48 \pm 0.08$ & $F: 0.57 \pm 0.07$ & F: .07 \\
\hline quadriceps femoris strength $(\mathrm{kg})$ & $6.1 \pm 1.9$ & $5.5 \pm 2.2$ & $5.2 \pm 1.5$ & .48 \\
\hline biceps brachii strength $(\mathrm{kg})$ & $6.3 \pm 2.2$ & $6.6 \pm 2.7$ & $6.9 \pm 1.1$ & .77 \\
\hline handgrip D $(\mathrm{kg})$ & $17.1 \pm 7.8$ & $19.9 \pm 7.3$ & $16.1 \pm 3.7$ & .47 \\
\hline handgrip D pred $(\%)$ & $87 \pm 25$ & $105 \pm 25$ & $90 \pm 26$ & .25 \\
\hline handgrip ND (kg) & $15.5 \pm 7.5$ & $16.0 \pm 6.5$ & $14.1 \pm 2.6$ & .78 \\
\hline handgrip ND pred (\%) & $93 \pm 38$ & $97 \pm 34$ & $90 \pm 28$ & .92 \\
\hline $\operatorname{MIP}\left(\mathrm{cmH}_{2} \mathrm{O}\right)$ & $32.8 \pm 15.3$ & $41.3 \pm 23.8$ & $31.1 \pm 16.4$ & .41 \\
\hline MIP pred (\%) & $68 \pm 36$ & $83 \pm 40$ & $67 \pm 41$ & .57 \\
\hline $\operatorname{MEP}\left(\mathrm{cmH}_{2} \mathrm{O}\right)$ & $67.4 \pm 25.2$ & $69.1 \pm 30.9$ & $56.4 \pm 16.7$ & .42 \\
\hline MEP pred (\%) & $61 \pm 23$ & $62 \pm 21$ & $53 \pm 20$ & .57 \\
\hline MVV (L/min) & $33.2 \pm 9.4$ & $40.2 \pm 14.0$ & $29.7 \pm 14.0$ & .16 \\
\hline gait speed $(\mathrm{m} / \mathrm{s})$ & $0.6 \pm 0.2$ & $0.7 \pm 0.4$ & $0.7 \pm 0.0$ & .83 \\
\hline
\end{tabular}

Note. Values are presented as percentages and mean $\pm S D . \mathrm{CG}=$ control group; RMTG $=$ respiratory muscle training group; PMTG $=$ peripheral muscle training group; $\mathrm{M}=$ male; $\mathrm{F}=$ female; $\mathrm{BMI}=$ body mass index; $\mathrm{ASM}=$ appendicular skeletal muscle mass; $\mathrm{ht}=$ height; $\mathrm{wt}=$ weight $\mathrm{D}=\mathrm{dominant}$ side; $\mathrm{ND}=$ nondominant side; pred = percentage of the reference value; $\mathrm{MIP}=$ maximum static inspiratory pressure; $\mathrm{MEP}=$ maximum static expiratory pressure; MVV = maximal voluntary ventilation . *Statistically significant $(p \leq .05)$.

RM endurance. Changes in MVV were statistically significant in the CG, $t(12)=-3.22 ; p=.007$, showing a $19.6 \%$ reduction from pre- to postintervention, whereas no significant changes were detected for either of the intervention groups (RMTG and PMTG). However, ascending trend values of $8.8 \%$ were seen for MVV in the PMTG, $t(8)=1.80 ; p=.110$.

Between-group ANOVA showed significant differences for MVV $(p=.020)$. Post hoc analysis showed that changes in MVV for the PMTG and RMTG were greater than changes in the CG.

Physical performance. Gait speed remained unchanged from pre- to postintervention in the three groups.
Training load. Training loads in RMTG (Table 3) significantly improved during the training sessions both for absolute values, $F(11,220)=130.2 ; \quad p<.001 ; \quad \eta^{2}=.867, \quad$ and percentages, $F(11,220)=33.3 ; p<.001 ; \eta^{2}=.625$. Similar findings were obtained for PMTG (Table 3 ) in biceps brachii, absolute values: $F(11$, $55)=14.9 ; p=.005 ; \eta^{2}=.749$ and percentages: $F(11,55)=19.7$; $p=.002 ; \eta^{2}=0.797$, and quadriceps femoris, absolute values: $F(11,44)=50.6 ; p<.001 ; \eta^{2}=.927$ and percentages: $F(11,44)$ $=41.0 ; p<.001 ; \eta^{2}=.911$.

Adherence. A secondary analysis following the same statistical procedure and dropping the adherence cutoff to $66 \%$ (2 days/week) 


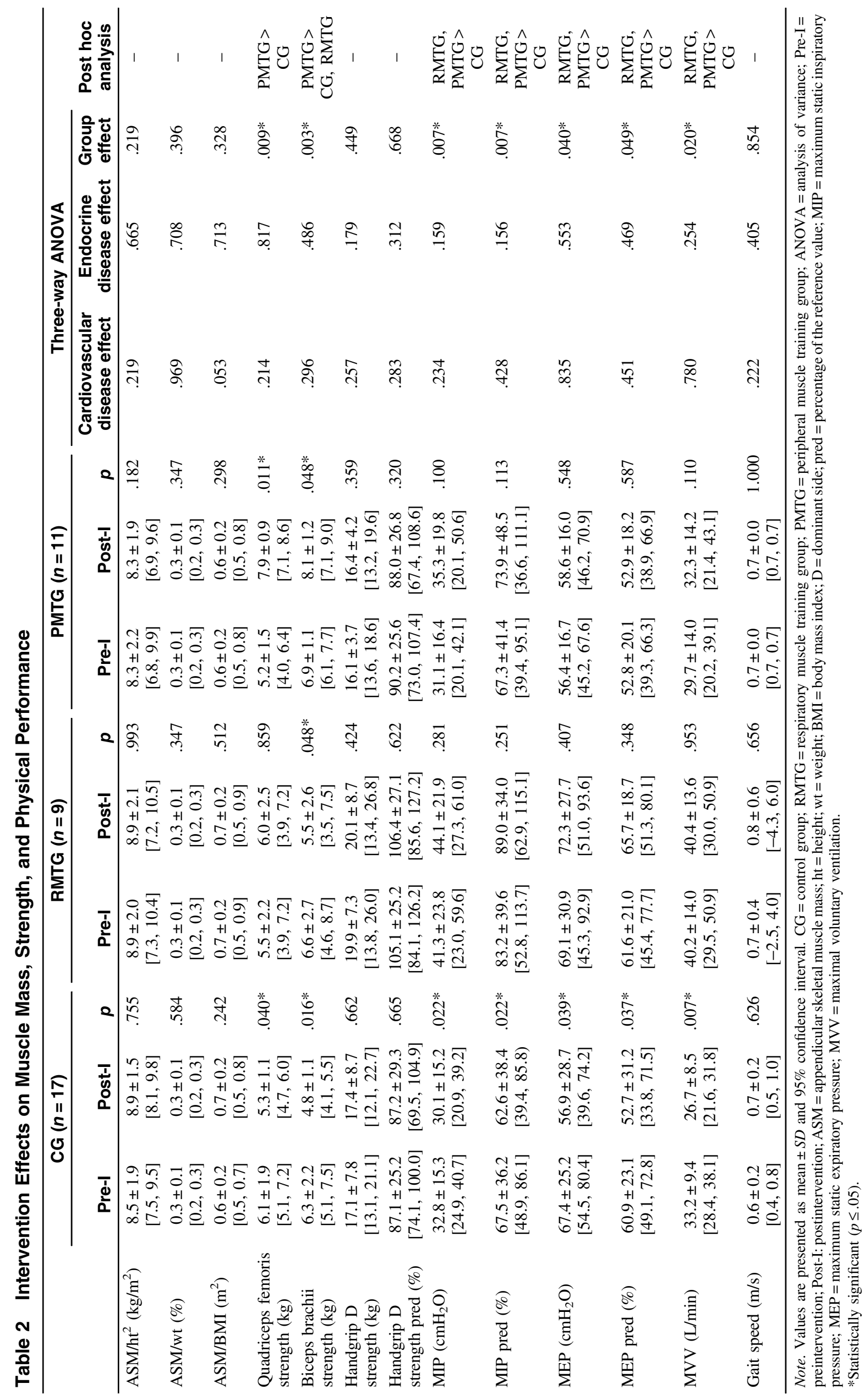


Table 3 Training Load for the RMTG and PMTG over the 12 Weeks of Training

\begin{tabular}{|c|c|c|c|c|c|c|}
\hline & \multirow{2}{*}{\multicolumn{2}{|c|}{$\begin{array}{c}\text { RMTG } \\
\text { Inspiratory muscles }\end{array}$}} & \multicolumn{4}{|c|}{ PMTG } \\
\hline & & & \multicolumn{2}{|c|}{ Biceps brachii } & \multicolumn{2}{|c|}{ Quadriceps femoris } \\
\hline & $\mathrm{MIP}\left(\mathrm{cmH}_{2} \mathrm{O}\right)$ & MIP (\%) & MIMS (kg) & MIMS (\%) & MIMS (kg) & MIMS (\%) \\
\hline Week 1 & $15.7 \pm 2.7$ & $43 \pm 12$ & $1.0 \pm 0.3$ & $20 \pm 2$ & $1.0 \pm 0.0$ & $19 \pm 1$ \\
\hline Week 2 & $18.6 \pm 2.8$ & $51 \pm 25$ & $1.3 \pm 0.5$ & $20 \pm 4$ & $1.5 \pm 0.3$ & $20 \pm 5$ \\
\hline Week 3 & $22.6 \pm 3.8$ & $61 \pm 30$ & $1.7 \pm 0.5$ & $21 \pm 5$ & $1.9 \pm 0.3$ & $23 \pm 6$ \\
\hline Week 4 & $24.9 \pm 5.1$ & $67 \pm 31$ & $2.5 \pm 0.9$ & $31 \pm 10$ & $2.8 \pm 0.7$ & $33 \pm 11$ \\
\hline Week 5 & $28.2 \pm 5.7$ & $76 \pm 41$ & $3.2 \pm 1.1$ & $39 \pm 10$ & $3.6 \pm 0.7$ & $42 \pm 13$ \\
\hline Week 6 & $29.4 \pm 5.8$ & $81 \pm 48$ & $3.7 \pm 1.5$ & $44 \pm 14$ & $4.7 \pm 0.5$ & $55 \pm 12$ \\
\hline Week 7 & $32.4 \pm 5.8$ & $90 \pm 54$ & $3.7 \pm 1.7$ & $44 \pm 18$ & $5.4 \pm 0.7$ & $63 \pm 13$ \\
\hline Week 8 & $34.2 \pm 7.2$ & $96 \pm 62$ & $4.0 \pm 1.7$ & $49 \pm 16$ & $6.4 \pm 1.1$ & $75 \pm 19$ \\
\hline Week 9 & $36.0 \pm 6.1$ & $100 \pm 61$ & $4.5 \pm 1.9$ & $54 \pm 18$ & $7.1 \pm 1.4$ & $82 \pm 20$ \\
\hline Week 10 & $36.6 \pm 6.1$ & $102 \pm 61$ & $5.0 \pm 2.1$ & $60 \pm 20$ & $1.7 \pm 1.5$ & $89 \pm 20$ \\
\hline Week 11 & $37.7 \pm 5.6$ & $105 \pm 62$ & $5.2 \pm 2.3$ & $62 \pm 20$ & $7.5 \pm 1.4$ & $88 \pm 21$ \\
\hline Week 12 & $37.7 \pm 5.5$ & $105 \pm 62$ & $5.4 \pm 2.3$ & $64 \pm 20$ & $7.8 \pm 1.6$ & $91 \pm 25$ \\
\hline
\end{tabular}

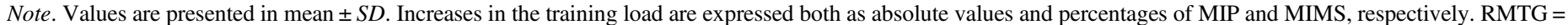

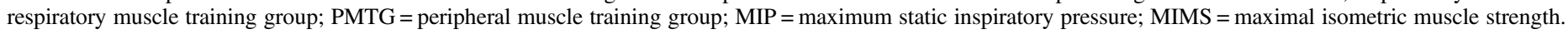

was performed to check the potential effectiveness of the intervention in this special setting (nursing home) and the characteristics of the exercise program. The new sample added four participants (three women and one man; three from RMTG and one from PMTG). Findings regarding both baseline characteristics and intervention effects on the main outcomes remained similar to previous analyses. Statistical significance was found in the same variables (quadriceps femoris strength, biceps brachii strength, MVV, and MIP and MEP [absolute and predicted values]), although post hoc analysis for quadriceps femoris strength showed greater changes for PMTG than RMTG, and RMTG in turn greater than CG (PMTG > RMTG > CG).

\section{Discussion}

This study revealed that resistance training mainly improved the skeletal muscle strength in institutionalized older people, although there were no significant changes in physical performance and muscle mass for both training groups. Therefore, the most relevant finding of this study remained counteracting the loss of muscle strength in this population. This result suggests that muscle strength was more sensitive to change, which declined significantly in the CG throughout the 3-month intervention. The improvement in muscle strength was more remarkable in the PMs (where there was a significant increase in the specifically trained group) than in the RMs. Moreover, there was no evidence to suggest that one specific muscle mass index (ASM/heigth ${ }^{2}$, ASM/weight, or ASM/ $\mathrm{BMI})$ was more appropriate than any other when assessing the effects of the 3-month resistance training program.

These results, as stated in a recent systematic review about sarcopenia by Cruf-Jentoft et al. (2014), reinforce the need to perform PM resistance training in older adults. For example, Binder et al. (2005) obtained an improvement in voluntary knee-extension force through progressive resistance training in frail older adults living in the community. In this study, the PM strength was assessed by maximal isometric quadriceps femoris and biceps brachii strength and also showed an improvement, although there was no significant effect seen in handgrip strength.
In this respect, a recent pilot study implemented in nursing home participants (Hassan et al., 2016) demonstrated an improvement in muscle strength (dominant handgrip measurement) as a consequence of a longer period of intervention ( 6 months), including balance training. Tieland, Verdijk, de Groot, and van Loon (2015) doubted if handgrip was a good measurement to detect changes in muscle strength derived from resistance training in frail older people because measurements could vary depending on age, sex, and nutrition, as also pointed out by Budziareck, Roig, and Barbosa (2008). In the present study, the improvement in quadriceps femoris and biceps brachii strength was important for two reasons: first, because there was a substantial reversion of the muscle strength loss in CG, and second because there was a significant increase in the PM trained group.

The present study demonstrated nonsignificant improvement in RM functioning in the RMTG. However, when compared with the CG values, there was a significant improvement. This result suggests that RM training has a preventive function, as RM values were maintained in older adults with sarcopenia who are institutionalized. This is relevant, bearing in mind the high prevalence of RM strength decline in this group of the population (Simões et al., 2009, 2010). According to Bahat et al. (2014), the decline affects more inspiratory than expiratory muscle strength in older adults with sarcopenia. Another explanation for this nonsignificant improvement in RM functioning in the trained group could be the short program duration or the number of sessions (Cebrià $i$ Iranzo et al., 2013). Despite following recommendations for an inspiratory muscle training program (intensity, frequency, and duration) for chronic obstructive pulmonary patients proposed by Hill et al. (2010) and Gosselink et al. (2011), these have been insufficient for the characteristics of the study population. Moreover, current recommendations for resistance training in older adults with sarcopenia (Cruz-Jentoft et al., 2014) point out that the time of intervention should be at least 3 months.

As has been reported in the Results section, the increase in training load was observed for both inspiratory muscle strength (MIP) and PM strength (maximal isometric muscle strength of biceps brachii and quadriceps femoris). This shows that there was a 
gain of workload training during the intervention. Moreover, this increase exceeded the percentage of load that was considered high enough to achieve training adaptations. Although the planning contemplated training at loads between $40 \%$ and $60 \%$, the participants trained with the loads they were able to lift.

The current study revealed a maintenance of gait speed after training as was also demonstrated in the study carried out by Hassan et al. (2016). A possible explanation could be that the training program did not include specific exercises to improve mobility, such as aerobic training and balance reeducation. Hassan et al. combine resistance and balance training without obtaining significant gain in the gait speed. Nevertheless, there was an improvement in standing capacity.

As mentioned above, the evolution of the muscle mass indexes over time (pre- to postintervention) was similar in both the CG and the trained ones. That is, it remained relatively stable for all three groups. In this respect, a pilot study implemented in nursing home participants also obtained similar results (Hassan et al., 2016). Although the result in muscle mass after training was unexpected, the improvement of muscle strength is a positive result because this could help prevent disability in older adults who are institutionalized. Ivey et al. (2000) and Hassan et al. (2016) suggest that the resistance training might induce functional (consequence of neural and/or metabolic muscle responses) versus structural (muscle cross-sectional area) adaptations.

Sarcopenia is related to various factors, such as comorbidity, mobility disorders, and malnutrition, thus experts (EWGSOP, IWGSP, and AWGSP y el FNIH) recommend combining exercise and nutrition interventions. Recent literature reviews (Denison, Cooper, Sayer, \& Robinson, 2015; Yu, Khow, Jadczak, \& Visvanathan, 2016) highlight inconsistent results mainly due to heterogeneity of population (age, sex, anthropometrics, settings, etc.) and intervention characteristics (resistance vs. aerobic, diversity in nutrition supplementation, etc.). The majority of published trial findings were carried out on healthy older adults living in the community (Bell et al., 2017) and in older adults with sarcopenia (Rondanelli et al., 2016). In settings such as nursing homes or hospitals, there is still a need for studies of these combined interventions (Bauer, Kaiser, \& Sieber, 2008), and those studies which combine interventions like Carlsson et al. (2011) the results are inconsistent.

The EWGSOP (Cruz-Jentoft et al., 2010) defines sarcopenia as low muscle mass and/or both low muscle strength and low gait. Currently there is an important debate about muscle mass cutoff (Moon et al., 2016; Pagotto \& Silveira, 2014; Studenski et al., 2014) and whether it should be considered and adapted to older population characteristics and settings (Kim et al., 2016; Meng et al., 2015; Tyrovolas et al., 2015), to avoid the no diagnosis of older adults with sarcopenia. For this reason, this study has included different muscle mass parameters (ASM/height ${ }^{2}$, ASM/ weight, and ASM/BMI) as proposed by Tyrovolas et al. (2015). With regard to the characteristics of the studied population (Spanish, institutionalized, and obese, BMI $>30 \mathrm{~kg} / \mathrm{m}^{2}$ ), we considered the appropriate parameters to be those related to weight (ASM/ weight and ASM/BMI) instead of the one usually applied (ASM/ height $^{2}$ ). We assessed which of these parameters was more sensitive for showing improvement after resistance training in older adults with sarcopenia who are institutionalized, and the findings were unclear because all parameters behaved similarly.

This study has several limitations. The analyzed sample diminishes considerably with respect to the participants' characteristics (Table 1), such as high comorbidity and advanced age
( $M_{\text {age }}>80$ years), functional impairment, and residency in a nursing home. This loss in follow-up is common in other nursing home research studies (Hassan et al., 2016; Mody et al., 2008). We consider only the sarcopenia status for inclusion criteria and outcomes and not fragility status, which contemplates additional functional parameters. We do not include follow-up of maintenance of resistance training effects. Despite ASM being a variable used infrequently in previous clinical trials, making direct comparison with this trial difficult, we use ASM indexes proposed by Tyrovolas et al. (2015), which are specific cutoffs for Spanish population. Furthermore, ASM may be more appropriate for detecting possible changes in upper and lower limb muscles. Finally, this trial does not collect physical activity as outcome.

Nonetheless, this study has some distinctive strengths. We compare two resistance-training protocols: one in RMs and one in PMs, both skeletal muscles affected by sarcopenia syndrome. Following the current international debate on reviewing the definition of sarcopenia, we have used the criteria of Tyrovolas et al. (2015) for muscle mass measurement (ASM/BMI) specifically for older Spanish people. Finally, as a relevant clinical finding, the improvement of muscle strength may be associated with a lower risk of mortality as suggested by Newman et al. (2006). Along these lines, the improvement of PM strength, as well as the absence of loss of PM strength in the respiratory musculature, may lead to a lower risk of mortality.

Mainly this clinical trial shows an improvement in skeletal muscle strength in older adults with sarcopenia who are institutionalized. This benefit can be extended to the older population with sarcopenia in both the community and nursing homes. To achieve this, health professionals' collaboration may be necessary to inform, encourage, and supervise the training programs (health promotion programs in primary care and the improvement of rehabilitation programs in nursing homes).

\section{Conclusions}

This resistance training has demonstrated an improvement in skeletal muscle strength (respiratory muscle and peripheral muscle) in older adults with sarcopenia who are institutionalized, indicating its preventive character and the potential benefit in carrying out this training in older adults with presarcopenia who are institutionalized. However, there was no significant improvement in muscle mass, in spite of measuring it with different parameters to avoid sarcopenia infradiagnosis and detect the training effects appropriately. Consequently, there are two fundamental considerations rising from this study: first, the need to adapt the physical exercise program by taking into account the specificity of the population and the desired functional outcome (improvement in muscle strength, walking speed, transfers, etc.) and, second, the need to combine physical exercise intervention with nutritional supplementation.

Finally, regarding the debate about cutoffs, this study does not clarify which muscle mass parameter is the most appropriate for detecting changes after physical interventions. As a result, the discussion continues on how to propose interventions and how to measure their effects on sarcopenia in the institutionalized older adult population.

\section{Acknowledgments}

This study was supported by Universitat de València (UV-INV-AE14269704) and has no conflict of interest to declare. The authors thank the 
participation of residents and staff, and especially Mary Martínez Martínez, the Technical Manager of La Saleta nursing homes. In addition, we would like to thank $\mathrm{M}^{\mathrm{a}}$ José Ponce Darós for her research support in the data collection.

\section{References}

American Thoracic Society/European Respiratory Society. (2002). ATS/ ERS Statement on respiratory muscle testing. American Journal of Respiratory and Critical Care Medicine, 166, 518-624. doi:10.1164/ recm.166.4.518

Bahat, G., Tufan, A., Ozkaya, H., Tufan, F., Akpinar, T.S., Akin, S., \& Karan, M.A. (2014). Relation between hand grip strength, respiratory muscle strength and spirometric measures in male nursing home participants. The Aging Male, 17, 136-140. PubMed ID: 24993454 doi:10.3109/13685538.2014.936001

Bauer, J.M., Kaiser, M.J., \& Sieber, C.C. (2008). Sarcopenia in nursing home participants. Journal of American Medical Directors Association, 9, 545-551. doi:10.1016/j.jamda.2008.04.010

Bean, J.F., Kiely, D.K., Herman, S., Leveille, S.G., Mizer, K., Frontera, W.R., \& Fielding, R.A. (2002). The relationship between leg power and physical performance in mobility-limited older people. Journal of American Geriatric Society, 50, 461-467. doi:10.1046/j.1532-5415. 2002.50111.x

Beaudart, C., Zaaria, M., Pasleau, F., Reginster, J.Y., \& Bruyère, O. (2017). Health outcomes of sarcopenia: A systematic review and meta-analysis. PLoS ONE, 12, e0169548. PubMed ID: 28095426 doi:10.1371/journal.pone.0169548

Bell, K.E., Snijders, T., Zulyniak, M., Kumbhare, D., Parise, G., Chabowski, A., \& Phillips, S.M. (2017). A whey protein-based multiingredient nutritional supplement stimulates gains in lean body mass and strength in healthy older men: A randomized controlled trial. PLoS ONE, 12, e0181387. PubMed ID: 28719669 doi:10.1371/ journal.pone.0181387

Binder, E.F., Yarasheski, K.E., Steger-May, K., Sinacore, D.R., Brown, M., Schechtman, K.B., \& Holloszy, J.O. (2005). Effects of progressive resistance training on body composition in frail older adults: Results of a randomized, controlled trial. The Journals of Gerontology, Series A: Biological Sciences \& Medicine Sciences, 60, 14251431. doi:10.1093/gerona/60.11.1425

Black, L.F., \& Hyatt, R.E. (1969). Maximal respiratory pressures: Normal values and relationship to age and sex. The American Review of Respiratory Disease, 99, 696-702. PubMed ID: 5772056

Borg, G. (2004). The Borg CR10 Scale folder. A method for measuring intensity of experience. Hasselby, Sweden: Borg Perception.

Budziareck, M.B., Roig, R., \& Barbosa, P. (2008). Reference values and determinants for handgrip strength in healthy subjects. Clinical Nutrition, 27, 357-362. PubMed ID: 18455840 doi:10.1016/j.clnu. 2008.03.008

Carlsson, M., Littbrand, H., Gustafson, Y., Lundin-Olsson, L., Lindelöf, N., Rosendahl, E., \& Håglin, L. (2011). Effects of high-intensity exercise and protein supplement on muscle mass in ADL dependent older people with and without malnutrition: A randomized controlled trial. Journal of Nutrition and Health Aging, 15, 554-560. doi:10. 1007/s12603-011-0017-5

Cebrià i Iranzo, M.A., Arnall, D.A., Igual Camacho, C., Tomás, J.M., \& Meléndez, J.C. (2013). Physiotherapy intervention for preventing the respiratory muscle deterioration in institutionalized older women with functional impairment. Archivos de Bronconeumología, 49, 1-9. PubMed ID: 29622961
Chen, K.K., Liu, L.K., Woo, J., Assantachai, P., Auyeung, T.W., Bahyah, K.S., \& Suzuki, T. (2014). Sarcopenia in Asia: Consensus report of the Asian Working Group for Sarcopenia. Journal of the American Medical Directors Association, 15, 95-101. PubMed ID: 24461239 doi:10.1016/j.jamda.2013.11.025

Cruz-Jentoft, A.J., Baeyens, J.P., Bauer, J.M., Boirie, Y., Cederholm, T., Landi, F., ... European Working Group on Sarcopenia in Older People. (2010). Sarcopenia: European consensus on definition and diagnosis: Report of the European Working Group on Sarcopenia in Older People. Age and Ageing, 39, 412-423. PubMed ID: 20392703 doi:10.1093/ageing/afq034

Cruz-Jentoft, A.J., Landi, F., Schneider, S.M., Zúñiga, C., Arai, H., Boirie, Y., \& Cederholm, T. (2014). Prevalence of and interventions for Sarcopenia in ageing adults: A systematic review. Report of the International Sarcopenia Initiative (EWGSOP and IWGS). Age and Ageing, 43, 748-759. PubMed ID: 25241753 doi:10.1093/ageing/ afu115

Cruz-Jentoft, A.J., Triana, F.C., Gómez-Cabrera, M.C., López-Soto, A., Masanés, F., Martín, P.M., \& Formiga, F. (2011). The emergent role of sarcopenia: Preliminary report of the Observatory of Sarcopenia of the Spanish Society of Geriatrics and Gerontology. Revista Española de Geriatría y Gerontología, 46, 100-110. PubMed ID: 21216498 doi:10.1016/j.regg.2010.11.004

Denison, H.J., Cooper, C., Sayer, A.A., \& Robinson, S.M. (2015). Prevention and optimal management of Sarcopenia: A review of combined exercise and nutrition interventions to improve muscle outcomes in older people. Clinical Interventions in Aging, 10, 859869. PubMed ID: 25999704

Enright, P.L., Kronmal, R.A., Manolio, T.A., Schenker, M.B., \& Hyatt, R.E. (1994). Respiratory Muscle strength in the older adult. Correlates and references values. Cardiovascular Health Study Research Group. American Journal of Respiratory Critical Care Medicine, 149, 430-438. PubMed ID: 8306041 doi:10.1164/ajrccm.149.2. 8306041

Ethgen, O., Beaudart, C., Buekinx, F., Bruyere, O., \& Reginster, J.Y. (2016). The future prevalence of Sarcopenia in Europe: A claim for public health action. California Tissue International, 100, 229-234. doi:10.1007/s00223-016-0220-9

Faul, F., Erdfelder, E., Lang, A.G., \& Buchner, A. (2007). G*Power 3: A flexible statistical power analysis program for the social, behavioral, and biomedical sciences. Behavior Research Methods, 39, 175-191. PubMed ID: 17695343 doi:10.3758/BF03193146

Gosselink, R., De Vos, J., van den Heuvel, S.P., Segers, J., Decramer, M., \& Kwakkel, G. (2011). Impact of inspiratory muscle training in patients with COPD: What is the evidence? European Respiratory Journal, 37, 416-425. PubMed ID: 21282809 doi:10.1183/ 09031936.00031810

Hassan, B.H., Hewitt, J., Keogh, J.W., Bermeo, S., Duque, G., \& Henwood, T.R. (2016). Impact of resistance training on Sarcopenia in nursing care facilities. A pilot study. Geriatric Nursing, 37, 116121. PubMed ID: 26694694 doi:10.1016/j.gerinurse.2015.11.001

Henwood, T.R., Keogh, J.W., Reid, N., Jordan, W., \& Senior, H.E. (2014). Assessing sarcopenic prevalence and risk factors in residential aged care: Methodology and feasibility. Journal of Cachexia, Sarcopenia and Muscle, 5, 229-236. PubMed ID: 24737112 doi:10.1007/ s13539-014-0144-z

Hill, K., Cecins, N.M., Eastwood, P.R., \& Jenkins, S.C. (2010). Inspiratory muscle training for patients with chronic obstructive pulmonary disease: A practical guide for clinicians. Archives of Physical Medicine and Rehabilitation, 91, 1466-1470. PubMed ID: 20801269 doi:10.1016/j.apmr.2010.06.010 
Ivey, F.M., Tracy, B.L., Lemmer, J.T., NessAiver, M., Metter, E.J., Fozard, J.L., \& Hurley, B.F. (2000). Effects of strength training and detraining on muscle quality: Age and gender comparisons. The Journals of Gerontology, Series A: Biological Sciences \& Medicine Sciences, 55, B152-B157; discussion B158-B159. doi:10.1093/ gerona/55.3.B152

Kim, K.M., Jang, H.C., \& Lim, S. (2016). Differences among skeletal muscle mass indices derived from height-, weight-, and body mass index-adjusted models in assessing Sarcopenia. Korean Journal of Internal Medicine, 31, 643-650. PubMed ID: 27334763 doi:10.3904/ kjim.2016.015

Landi, F., Liperoti, R., Fusco, D., Mastropaolo, S., Quattrociocchi, D., Proia, A., \& Onder, G. (2012). Prevalence and risk factors of Sarcopenia among nursing home older residents. The Journals of Gerontology, Series A: Biological Sciences \& Medicine Sciences, 67A, 48-55. doi:10.1093/gerona/glr035

Lobo, A., Saz, P., \& Marcos, G. (2002). Adaptation of the Mini-Mental State Examination (MMSE). Madrid, Spain: TA Ediciones.

Luna-Heredia, E., Martín-Peña, G., \& Ruiz-Galiana, J. (2005). Handgrip dynamometry in healthy adults. Clinical Nutrition, 24, 250-258. PubMed ID: 15784486 doi:10.1016/j.clnu.2004.10.007

Meng, N.H., Li, C.I., Liu, C.S., Lin, W.Y., Lin, C.H., Chang, C.K., \& Lin, C.C. (2015). Sarcopenia defined by combining height-and weightadjusted skeletal muscle indices is closely associated with poor physical performance. Journal of Aging and Physical Activity, 23, 597-606. PubMed ID: 25562330 doi:10.1123/japa.2014-0036

Mody, L., Miller, D.K., McGloin, J.M., Freeman, M., Marcantonio, E.R., Magaziner, J., \& Studenski, S. (2008). Recruitment and retention of older adults in aging research. Journal of American Geriatrics Society, 56, 2340-2348. doi:10.1111/j.1532-5415.2008.02015.x

Moon, J.H., Kim, K.M., Kim, J.H., Moon, J.H., Choi, S.H., Lim, S., \& Jang, H.C. (2016). Predictive values of the New Sarcopenia Index by the Foundation for the National Institutes of Health Sarcopenia Project for Mortality among Older Korean Adults. PLOS ONE, 11, e0166344. PubMed ID: 27832145 doi:10.1371/journal.pone.0166344

National Institutes of Health. (1996). Bioelectrical impedance analysis in body composition measurement: National Institutes of Health Technology Assessment Conference Statement. American Journal of Clinical Nutrition, 64, 524S-532S. doi:10.1093/ajcn/64.3.524S

Neder, J.A., Andreoni, S., Lerario, M.C., \& Nery, L.E. (1999). Reference values for lung function tests. II. Maximal respiratory pressures and voluntary ventilation. Brazilian Journal of Medical and Biological Research, 32, 719-727. PubMed ID: 10412550 doi:10.1590/S0100879X1999000600007

Newman, A.B., Kupelian, V., Visser, M., Simonsick, E., Goodpaster, B., Nevitt, M., \& Harris, T.B. (2003). Health ABC Study Investigators. Sarcopenia: Alternative definitions and associations with lower extremity function. Journal of American Geriatrics Society, 51, 1602-1609. doi:10.1046/j.1532-5415.2003.51534.x

Newman, A.B., Kupelian, V., Visser, M., Simonsick, E.M., Goodpaster, B.H., Kritchevsky, S.B., \& Harris, T.B. (2006). Strength, but not muscle mass, is associated with mortality in the health, aging and body composition study cohort. The Journals of Gerontology, Series A: Biological Sciences \& Medical Sciences, 61, 72-77. PubMed ID: 16456196

Pagotto, V., \& Silveira, E.A. (2014). Methods, diagnostic criteria, cutoff points, and prevalence of Sarcopenia among older people. Scientific World Journal, 2014, 1-11. PubMed ID: 25580454 doi:10.1155/ 2014/231312
Rondanelli, M., Klersy, C., Terracol, G., Talluri, J., Maugeri, R., Guido, D., \& Perna, S. (2016). Whey protein, amino acids, and vitamin D supplementation with physical activity increases fat-free mass and strength, functionality, and quality of life and decreases inflammation in sarcopenic older adult. American Journal of Clinical Nutrition, 103, 830-840. PubMed ID: 26864356 doi:10.3945/ajcn. 115.113357

Rubbieri, G., Mossello, E., \& Di Bari, M. (2014). Techniques for the diagnosis of sarcopenia. Clinical Cases Mineral Bone Metabolism, 11, 181-184. PubMed ID: 25568650

Salvà, A., Serra-Rexach, J.A., Artazad, I., Formigae, F., Rojano, X., Cuestaf, F., \& Cruz-Jentoftl, A.J. (2016). Prevalence of sarcopenia in Spanish nursing homes: Comparison of the results of the ELLI study with other populations.. Revista Española Geriatría y Gerontología, 51, 260-264. doi:10.1016/j.regg.2016.02.004

Simões, L.A., Dias, J.M., Marinho, K.C., Pinto, C.L., \& Britto, R.R. (2010). Relationship between functional capacity assessed by walking test and respiratory and lower limb muscle function in community-dwelling elders. Revista Brasileira de Fisioterapia, 14, 24-30. doi:10.1590/S1413-35552010000100005

Simões, R.P., Castello, V., Auad, M.A., Dionísio, J., \& Mazzonetto, M. (2009). Prevalence of reduced respiratory muscle strength in institutionalized older adult people. Sao Paulo Medical Journal, 127, 78-83. doi:10.1590/S1516-31802009000200005

Studenski, S.A., Peters, K.W., Alley, D.E., Cawthon, P.M., McLean, R.R., Harris, T.B., \& Vassileva, M.T. (2014). The FNIH Sarcopenia project: Rationale, study description, conference recommendations, and final estimates. The Journals of Gerontology, Series A: Biological Sciences \& Medicine Sciences, 69, 547-558. doi:10.1093/gerona/ glu010

Tieland, M., Verdijk, L.B., de Groot, L.C., \& van Loon, L.J. (2015). Handgrip strength does not represent an appropriate measure to evaluate changes in muscle strength during an exercise intervention program in frail older people. International Journal of Sport Nutrition and Exercise Metabolism, 25, 27-36. PubMed ID: 24903908 doi:10. 1123/ijsnem.2013-0123

Tilson, J.K., Sullivan, K.J., Cen, S.Y., Rose, D.K., Koradia, C.H., Azen, S.P., ... Locomotor Experience Applied Post Stroke (LEAPS) Investigative Team. (2010). Meaningful gait speed improvement during the first 60 days poststroke: Minimal clinically important difference. Physical Therapy, 90, 196-208. doi:10.2522/ptj. 20090079

Tyrovolas, S., Koyanagi, A., Olaya, B., Ayuso-Mateos, J.L., Miret, M., Chatterji, S., \& Haro, J.M. (2015). The role of muscle mass and body fat on disability among older adults: A cross-national analysis. Experimental Gerontology, 69, 27-35. PubMed ID: 26048566 doi:10.1016/j.exger.2015.06.002

Wei-Ju, L., Li-Kuo, L., Li-Ning, P., Ming-Hsien, L., Liang-Kung, C., \& ILAS Research Group. (2013). Comparisons of Sarcopenia defined by IWGS and EWGSOP criteria among older people: Results from the I-Lan longitudinal aging study. Journal of the American Medical Directors Association, 14, 528.e1-528.e7. PubMed ID: 23664768 doi:10.1016/j.jamda.2013.03.019

Yu, S.C., Khow, K.S., Jadczak, A.D., \& Visvanathan, R. (2016). Clinical screening tools for sarcopenia and its management. Current Gerontology and Geriatrics Research, 2016, 1-10. PubMed ID: 26966433 doi:10.1155/2016/5978523 